\title{
Correlates of Parent-Child Physiological Synchrony and Emotional Parenting: Differential Associations in Varying Interactive Contexts
}

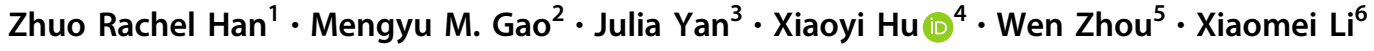

Published online: 13 February 2019

(c) The Author(s) 2019

\begin{abstract}
Objectives Parent-child synchrony during interaction might possess important features that underlie parenting processes throughout development. However, little is known regarding the association between parent-child physiological synchrony and emotional parenting behaviors during middle childhood. The main goal of the study was to examine whether emotional parenting was positively or negatively associated with parent-child physiological synchrony for school-age children.

Methods Adopting a biopsychosocial perspective, we incorporated the interbeat interval (IBI) and behavioral observation data of 150 parent-child dyads (child $M$ age $=8.77, S D=1.80$ ) to explore the patterns of moment-to-moment dyadic physiological synchrony and to investigate whether these patterns were associated with two emotional parenting behaviors (psychological control and psychological unavailability).

Results Our findings provided some initial evidence that in low to moderately stressful situations that mimic daily parentchild interaction, parent-child physiological synchrony was indicative of different emotional parenting behaviors in various parent-child interactive situations. Specifically, in the collaborative context (parent-child working together to complete a task), parent-child physiological synchrony was indicative of less psychological unavailability, whereas in the competitive context (parent-child resolving disagreement with each other), parent-child physiological synchrony was indicative of less psychological control. The study implications and future research directions are discussed.

Conclusions Overall, our findings suggested that dyadic physiological synchrony, indexed by parent-child moment-tomoment matching of IBI, was associated with fewer negative emotional parenting behaviors.
\end{abstract}

Keywords physiological synchrony $\cdot$ IBI $\cdot$ emotional parenting $\cdot$ psychological control $\cdot$ psychological unavailability

The last decade has witnessed a shift in parenting research such that the field has become increasingly interested in the biopsychosocial perspective that encompasses not only the behavioral but also the physiological dimensions of parent-

Xiaoyi $\mathrm{Hu}$

huxiaoyi@bnu.edu.cn

1 Faculty of Psychology, Beijing Normal University, Beijing, China

2 Department of Psychology, University of Notre Dame, Notre Dame, USA

3 Department of Human Sciences, The Ohio State University, Columbus, $\mathrm{OH}$, USA

4 Faculty of Education, Beijing Normal University, Beijing, China

5 Department of Evolutionary Anthropology, Duke University, Durham, UK

6 Department of Human Development and Family Studies, University of Illinois at Urbana-Champaign, Champaign, IL, USA child dyads (Feldman 2012a, 2012b; Repetti et al. 2002). Conforming to this trend in parenting research, the current study considered both the physiological and behavioral aspects of parenting and examined whether emotional parenting behaviors captured in parent-child interaction were associated with moment-to-moment parent-child physiological synchrony for children in their middle childhood.

Synchrony has been proposed as a construct to capture the mutuality and reciprocity of dyadic interaction (Harrist and Waugh 2002). Parent-child synchrony has been frequently used to represent the quality of parent-child interactions by providing a concrete and continuous description of the overarching process that coordinates the exchanges of hormonal, behavioral, and physiological stimuli between parent and child during social contact. Dyadic synchrony occurs as early as in infancy with the emergence of symbolic exchanges between parent and child (Feldman 2012a). Over repeated family contact within day-to-day experience, each member of the bonding relationship develops 
appropriate sensitivities and responses to various cues during interaction (Fleming et al. 1999). This coordination of behavioral and biological cue-exchange processes leads to the formation of selective and enduring attachment (Feldman 2012a) and might continuously take effect on the child across development (Graham et al. 2017).

Although parent-child synchrony represents processes that incorporate both behavioral and biological stimuli, research used to predominantly focus on the behavioral aspects, such as joint attention, shared affect, and cooccurred body movement (e.g., Feldman and Eidelman 2004; for a review, see Harrist and Waugh 2002). Only recently have empirical studies begun to pay more attention to the physiological aspects of parent-child interaction, aiming to capture the synchronous characteristics of the dyadic exchange beyond the behavioral level (Suveg et al. 2016). Many indicators have been adopted to assess physiological synchrony. For example, mother-infant dyads showed coordinated heart rhythms during face-to-face interactions as early as during the first three months of the infant's life (Feldman et al. 2011). Respiratory sinus arrhythmia (RSA), another critical measure of vagal tone, was found to be coordinated between school-age children and their mothers (Woody et al. 2016). Regardless of which indicator is considered, physiological synchrony seems common in parent-child dyads during interaction across developmental stages and might have important implications for parenting (Feldman 2012a).

In infancy, parental behaviors, shaped by the parent's physiology and emotional states, are the only phenomenon directly available to the infants during interaction and thus are believed to predict parent-child physiological synchrony (Feldman 2012a). For this reason, researchers have suggested that parents' physiological systems can influence infants' biological organization of bonding with their parents through parenting (see Feldman 2012a for a review). Although physiological synchrony between parents and their children could occur at various stages of child development beyond infancy (Clearfield et al. 2014), few investigations have gone beyond infancy to target parenting and parent-child physiological synchrony in middle childhood (Woltering et al. 2015). However, children in their middle childhood are becoming more independent actors in parent-child interactions (Harrist and Waugh 2002), and we are curious about whether the parent still holds a critical role in the establishment of physiological synchrony between parent-child dyads. Due to the paucity of related research, many researchers have called for more research on this topic in older children to better understand how physiological synchrony operates and relates to parenting behaviors for children well beyond infancy (e.g., Suveg et al. 2016).

Unlike the clear association between parenting and parent-child behavioral synchrony for this age group (that positive behavioral synchrony is believed to function as a relationship builder and may motivate parents to engage in more positive parenting; Harrist and Waugh 2002), limited research has directly tested the link between emotional parenting behaviors and parent-child physiological synchrony, and the results have been largely mixed. Although no direct literature is available on the relationship between emotional parenting and parent-child physiological synchrony, several studies on parental characteristics and physiological synchrony suggested that positive family attributes seemed to be associated with greater parent-child physiological synchrony. For example, one study showed that higher socio-economic status (SES) strengthened parent-child physiological synchrony in infancy (Clearfield et al. 2014). Another study found the dyads without histories of maternal major depressive disorder (MDD) displayed more positive RSA concordance than their counterparts with maternal history of MDD during middle childhood (Woody et al. 2016). Additionally, a positive association was demonstrated between physiological synchrony and parental behaviors that might promote parentchild closeness during early adolescence (Papp et al. 2009).

Other studies, however, did not find parent-child physiological synchrony a positive indicator of adaptive parenting. For example, Suveg et al. (2016) demonstrated that greater parent-child physiological synchrony was related to more negative interactions between parents and preschoolers, such as emotional and communicative distance, especially under high-risk family contexts. Along a similar vein, Papp et al. (2009) found that parent-child cortisol synchrony was associated with more parental negative affect toward adolescents. These findings seemed to suggest that if one member of the dyad is under stress or experiencing a negative effect, the physiological matching between the parent-child dyads may confer additional risk for maladaptation, so that these dyads may be more likely to engage in negative behavioral interactions (Suveg et al. 2016). These contradictory findings warranted us to come back to a fundamental question on emotional parenting and physiological synchrony-what is the relationship between parent-child synchrony and emotional parenting, especially for children well beyond infancy?

Also, although differential indicators have been examined on parent-child physiological synchrony, it has been suggested that rather than investigating the averages of mother and child physiological functioning across specific time intervals (e.g., calculating physiological synchrony every $30 \mathrm{~s}$ ), it makes more sense to reply on moment-tomoment physiological measures as physiological synchrony takes place in such real-time fashion (Suveg et al. 2016). Interbeat interval (IBI) series data assessing temporal synchrony of autonomic reactivity allows the examination of parent-child physiological synchrony within each second 
and is considered a more appropriate measurement on this dyadic construct.

The main goal of the current study was to examine whether emotional parenting was positively or negatively associated with parent-child physiological synchrony for school-age children using moment-to-moment IBI measures. We targeted the moderate to low stressful situations, mimicking the daily interaction of the majority of parentchild dyads, and targeted the typical emotion-related parenting behaviors, such as psychological unavailability and psychological control. Specifically, we aim to explore the relationship between parenting and parent-child physiological synchrony in middle childhood, after controlling for parental and child gender, child age, and parental and child psychopathological symptoms.

\section{Method}

\section{Participants}

In total, 150 parent-child dyads (Total $N=300$ ) were recruited through flyers displayed in a local community of a major city in China. Participants consisted of school-age children between the ages of 6-12 (63 girls, $M_{\text {age }}=8.54$, $S D=1.67$ ) and their self-identified primary caregivers (121 biological mothers, 29 biological fathers, $M_{a g e}=$ $39.22, S D=4.07)$. In terms of family income, 114 families $(76.0 \%)$ in the sample have annual income higher than the average of the city (i.e., around \$18,500 annually; National Bureau of Statistics of the People's Republic of China, 2015). In terms of marital status, 142 parents (94.7\%) were currently married, and all parents identified themselves as the biological parents of the participating children. For parents' educational backgrounds, $6.0 \%$ of parents had high school training, $60.7 \%$ were college undergraduates, and $33.3 \%$ had attended graduate schools.

\section{Procedures}

Parents and children were informed of the purpose and procedure of the study and signed informed consent or minor assent forms upon arriving at the research laboratory. Before the interaction tasks, the parent-child dyads were instructed to sit relaxed and quietly to become acclimated to the experiment setting, where they were hooked up to physiological recorders and were given approximately 3 min to adapt to the equipment. ECG electrodes were attached to the participant's left leg $(+)$, left arm $(-)$, and right leg (ground), based on Einthoven's triangle (Lead III; Biopac Systems, Inc., 2016). The dyads then participated in a 2-min resting session where they were instructed to sit relaxed and breathe regularly without speaking or moving.
After this baseline period, the dyads were asked to participate in two interaction tasks. In one task, the dyads were asked to collaborate and draw a picture of a house and a tree in four minutes using Etch-A-Sketch. In the other task, the dyads were asked to discuss a mutually selected topic of conflict for four minutes. The dyads were asked to try to come up with a solution to their conflict. Dyads were compensated $180 \mathrm{RMB}$ (about \$30) for their participation, and children were given a small token of appreciation. All study procedures were conducted in accordance with the sponsoring university's Institutional Review Board.

\section{Measures}

\section{Physiological synchrony}

Physiological synchrony is indicated by a close match of the physiological states between parent-child dyads, as suggested by previous research (e.g., Suveg et al. 2016). As the nature of synchrony is a temporal process, time is a critical parameter (Rosenfeld et al. 1981). Interbeat interval (IBI) series is a recommended moment-to-moment indicator to measure the temporal synchrony of autonomic nervous reactivity (see Feldman et al. 2011; Suveg et al. 2016) and was thus used in the current study to indicate physiological synchrony. Because each individual's IBI series underwent autoregressive integrated moving average (ARIMA) modeling (Feldman et al. 2011), we followed previous research and applied this model to examine synchrony independent of the autocorrelation in each IBI series (Feldman et al. 2011).

\section{Parenting behaviors}

The emotional parenting behaviors of interest in this study included psychological unavailability and psychological control. Behavioral observations were coded using the scales described below, which were translated and adapted from previously developed observational codes (Minnesota Longitudinal Study of Parents and Children, n.d; Shaffer et al., n.d.). A group of trained researchers coded these observations independently (three coders on each video) and determined the final score for each scale through discussion. Inter-rate reliability was assessed by the $10 \%$ of the coded sample and calculating the intra-class correlation coefficients.

Psychological unavailability The emotional unavailability scale reflected the extent to which a parent failed to be aware of or respond appropriately to the child's emotional needs. Coders used a seven-point Likert scale from -3 to 3 . Parents who scored low (e.g., -3) on this scale exhibited sensitive awareness and appropriate responses to the child's 
emotional experience (e.g., labeling, validating, expressing curiosity or empathy to the child's emotions, being able to comfort a distressed child). Parents who scored high (e.g., 3 ) on this scale appeared to be unavailable to the child's emotional needs or detached from the interaction (e.g., ignoring or showing impatience towards the child's emotional expressions or bids for attention, not being able to comfort a distressed child or share the child's positive experience, only interacting with the child when necessary or required to interact by the task). The inter-rater reliability for emotional unavailability was .90 .

Psychological control The psychological control scale measured the extent to which the parent failed to recognize the child's individuality and attempted to control the child's opinions, ideas, and feelings. It was coded on a seven-point Likert scale. Parents who scored low (e.g., 1) on this scale did not show any attempt to coerce or squash the child's own ideas, opinions, and feelings, whereas parents who scored high (e.g., 7) on this scale attempted to align the child with their own perspective through psychological controlling approaches (e.g., eliciting of guilt or shame, derision, coercion, and threats of love withdrawal). Salient indicators of psychological control also included intrusive interruptions when the child was speaking and pointing out the inadequacy of the child's opinions without explaining or offering alternative options. The inter-rater reliability for psychological control was .89 .

\section{Control variables}

The control variables for this investigation included basic demographic variables (i.e., child age, child and parent sex) as well as parent and child psychopathological symptoms due to their potential correlates with parent-child physiological synchrony (e.g., Lunkenheimer et al. 2017). Parent psychopathological symptoms were measured by Symptom Checklist-90-Revised (SCL-90, Derogatis, 1994), a 90-item questionnaire that evaluates the psychopathological symptoms an adult has experienced over the last 7 days. The tscore of the sums of all items (the t-score of the Global Severity Index) was used for the current study, and increasing scores indicate increasing psychological distress. The Chinese version of the SCL-90 has been widely used (Zhang 2005) and has shown good test-retest reliabilities and validity. For the present study, Cronbach's alpha of the total scale was .98. Child psychopathological symptoms were assessed by the Child Behavior Checklist (CBCL; Achenbach, 1991). The CBCL is a 118-item measure of parent-reported psychosocial functioning of a child over the past 6 months that yields a total score reflecting the overall emotional and behavioral problems of the child. The Chinese version of the CBCL has been used widely (e.g.,
Crijnen et al. 1999; Poston and Falbo 1990), and it has exhibited good test-retest reliability and validity (Leung et al. 2006). The reliability of the overall scale for the current study was good; Cronbach's alpha was .92.

\section{Data Analyses}

To calculate the physiological synchrony of the parent and child dyads and eliminate the autocorrelation in each IBI series, we conducted autoregressive integrated moving average (ARIMA) modeling (see Suveg et al. 2016 for more details). To conduct autoregressive integrated moving average (ARIMA) modeling, both members of each dyad were required to have the same amount of IBI epochs. Thus, we calculated $4 \mathrm{~Hz}$ mean IBI series using MindWare, via resampling at fixed intervals with interpolation (Berger et al. 1986).

SPSS Expert Modeler was used to calculate and identify the best-fitting ARIMA model for each IBI series. All data were reviewed for artifacts and corrected manually if artifacts led to the artificial detection of $\mathrm{R}$ peaks. Following the modeling procedures, the residual series of each ARIMA model were obtained and used to calculate the crosscorrelation functions (CCFs) with no time lag for each parent-child dyad. The CCFs are used to measure the physiological concordance of each dyad beyond individual internal rhythms (Feldman et al. 2011). Then, a series of correlational analyses as well as one-way ANOVA tests were performed to explore whether demographic and control variables were significantly associated with any study variables. Lastly, the linear regression analyses predicting parent-child physiological synchrony from emotional parenting behaviors for each task were adopted to examine whether the parent-child physiological synchrony of each task could be explained by the emotional parenting behaviors, even after controlling for potential covariates.

\section{Results}

Descriptive statistics and correlations among the study and control variables are presented in Table 1. Based on the correlational results, child age was significantly and negatively associated with child overall behavioral problems, as well as with parent-child physiological synchrony and parental control during the conflict discussion task but not during the Etch-A-Sketch task. However, the parent-child physiological synchrony of the Etch-A-Sketch task was significantly and negatively associated with parents' psychological unavailability in that task. However, the similar patterns were not evident in the conflict discussion task; no significant correlation was found between parent-child physiological synchrony and parenting behaviors in this 
Table 1 Descriptive statistics and correlations among study and control variables

\begin{tabular}{|c|c|c|c|c|c|c|c|c|c|c|c|c|c|c|}
\hline & $\mathrm{N}$ & M & SD & 1 & 2 & 3 & 4 & 5 & 6 & 7 & 8 & 9 & 10 & 11 \\
\hline 1. Child sex ${ }^{a}$ & 150 & .42 & .50 & & & & & & & & & & & \\
\hline 2. Child age & 150 & 8.54 & 1.67 & .00 & & & & & & & & & & \\
\hline 3. Parent $\operatorname{sex}^{b}$ & 150 & .19 & .40 & -.07 & $.15^{\dagger}$ & & & & & & & & & \\
\hline 4. ES_IBI & 130 & -.00 & .04 & -.08 & .09 & -.07 & & & & & & & & \\
\hline 5. ES_PU & 146 & -.77 & 1.22 & .01 & -.10 & -.04 & $-.18 *$ & & & & & & & \\
\hline 6. ES_PC & 146 & 1.42 & .79 & -.12 & -.04 & -.09 & -.12 & $.49 * *$ & & & & & & \\
\hline 7. Con_IBI & 127 & .01 & .05 & -.04 & $-.24 * *$ & -.06 & .03 & -.14 & -.07 & & & & & \\
\hline 8. Con_PU & 150 & -.68 & 1.32 & -.04 & -.03 & .00 & .01 & $.50 * *$ & $.22 *$ & -.03 & & & & \\
\hline 9. Con_PC & 150 & 2.58 & 1.43 & .03 & $-.16^{*}$ & $-.20 *$ & .00 & $.28 * *$ & $.33 * *$ & -.11 & $.33 * *$ & & & \\
\hline 10. SCL_90 & 150 & 50.92 & 11.60 & .11 & -.04 & -.06 & .03 & -.04 & -.06 & .14 & -.02 & $.20^{*}$ & .05 & \\
\hline 11. CBCL_T & 149 & 56.48 & 9.70 & .06 & $-.18 *$ & $-.18 *$ & .05 & .06 & -.15 & .03 & .06 & .20 & .02 & $.35^{* *}$ \\
\hline
\end{tabular}

Note: $P U$ psychological unavailability, $P C$ psychological control, ES Etch-A-Sketch, Con conflict discussion

${ }^{\dagger} p<.1,{ }^{*} p<.05,{ }^{* *} p<.01$

${ }^{\mathrm{a}}$ Child sex was coded as 0 (male) and 1 (female)

${ }^{\mathrm{b}}$ Parent sex was coded as 0 (male) and 1 (female)

task. Additionally, parental psychopathological symptoms were not significantly associated with any study variable except for child overall behavioral problems and parental psychological control in the conflict discussion task, with parents who reported more psychopathological symptoms reporting more behavioral problems of their children and displaying more psychologically controlling behaviors when trying to resolve a conflict with their child. One-way ANOVA tests examining the relationship between parent/ child sex and all study variables showed that parent or child sex was not significantly associated with any study variable except parental psychological control in the conflict discussion task, with fathers displaying more controlling behaviors, $\mathrm{F}(1,148)=6.08, p=.02$.

We then tested whether physiological synchrony was explained by emotional parenting behaviors during the EtchA-Sketch and the conflict discussion tasks, controlling for the significant covariates indicated in the correlational analyses. The results of the linear regression analyses showed that, in the Etch-A-Sketch task, parental psychological unavailability significantly predicted parent-child physiological synchrony, $\beta=-.18, \mathrm{t}(128)=-2.02, p=.045$. Parental psychological unavailability also explained a significant proportion of variance of physiological synchrony in this task, $\mathrm{R}^{2}=.18, \mathrm{~F}(1$, $128)=4.09, p=.045$. However, parental psychological control did not significantly predict parent-child physiological synchrony, $\beta=-.12, \mathrm{t}(128)=-1.32, p=.19$. In the conflict discussion task, parental psychological unavailability did not significantly predict parent-child physiological synchrony, after controlling for the significant covariate (i.e., child age), $\beta=.10, \mathrm{t}(124)=.94, p=.350$, but child age remained a significant predictor in the model, $\beta=-.24, \mathrm{t}(124)=-2.73$, $p=.007$. Parental psychological control significantly predicted parent-child physiological synchrony, $\beta=-.19$, $\mathrm{t}(126)=-2.09, p=.039$, even after controlling for the significant covariates (i.e., child age, parent sex, and parental psychopathological symptoms). Except for parent sex, parental psychopathological symptoms $(\beta=.15, \mathrm{t}(126)=1.76$, $p=.081)$ and child age $(\beta=-.26, \mathrm{t}(126)=-2.92, p=.004)$ remained significant predictors in the model, together explaining a significant proportion of the variance of physiological synchrony in this task, $\mathrm{R}^{2}=.32, \mathrm{~F}(1,125)=3.58$, $p=.008$.

\section{Discussion}

Parent-child synchrony during interaction might possess important features that underlie parenting processes throughout child development (Feldman 2012b). Little research has examined whether parent-child physiological synchrony during low to moderately stressful family interaction reflects how well parents respond to their children's emotional needs during middle childhood. Adopting a biopsychosocial perspective, this study examined the direct association between parent-child physiological synchrony and emotional parenting behaviors observed during two interaction tasks that mimic typical daily interaction of parent-child dyads. One task (i.e., Etch-A-Sketch) required the collaboration of the parent-child, and the other (i.e., conflict discussion) was characterized by potential competition between the dyads.

Overall, our findings suggested that dyadic physiological synchrony, indexed by parent-child moment-to-moment 
matching of IBI, was associated with fewer negative emotional parenting behaviors. These results provided some evidence that parents whose physiological states better match those of their children during interaction engage in fewer emotionally maltreating behaviors such as psychological availability and psychological control (Shaffer et al. 2009). Previous studies preliminarily demonstrated the contexts under which parent-child physiological synchrony was presented (Feldman 2007; Williams et al. (2013)) with limited attention to how such physiologically concordant patterns were associated with specific parenting behaviors. Our findings added to the knowledge on this line of research and were generally consistent with findings on parent-child behavioral synchrony suggesting that synchrony was a positive indicator of parenting (Harrist and Waugh 2002). However, we found that such physiological synchrony was associated with different parenting behaviors in varying parent-child interactive contexts.

Specifically, there was a clear negative association between parent-child physiological synchrony and parental psychological unavailability but not psychological control in the collaboration task, suggesting that parents who seemed to be more physiologically synchronous with their children were less emotionally detached from their children during family interactions that required collaboration. In contrast, there was a clear negative association between parent-child physiological synchrony and parental psychological control but not psychological unavailability in the competition task, suggesting that parents who seemed to be more physiologically synchronous with their children adopted fewer psychologically controlling behaviors when trying to reach a resolution with their child.

These results might be explained from the nature of differential patterns of interaction and the meanings of various emotional parenting behaviors. Despite the resemblance between psychological unavailability and psychological control in terms of the valence of parenting behaviors, psychological control and psychological unavailability are two conceptually distinct constructs describing different emotional parenting behaviors. Psychological unavailability might apply more to interactive situations where children need parents' emotional assistance to finish a challenging task, and the emotionally unavailable parents might be less likely to provide assistance. In contrast, psychological control might apply more to the interactive situations with a goal of achieving parent-child agreement, when the controlling parents might be more likely to invalidate children's ideas and manipulate children's opinions during the process (Barber and Harmon 2002). Therefore, lack of parent-child physiological synchrony in the collaboration task might be more likely to reflect parents' emotional unavailability, whereas parent-child physiological synchrony in the conflict discussion task might be more likely to reflect parents' psychological control, both dependent on what types of emotional parenting the task demanded.

Since there was no prior investigation that specifically investigated the direction of the association between physiological synchrony and emotional parenting behaviors within a given task, we did not put forth any specific hypotheses. However, the results indicated that the interplay between parent-child physiological synchrony and emotional parenting behaviors might be more complex than expected, possibly depending on the nature of parent-child interaction. The findings also highlighted the importance of considering the contexts of parent-child interaction when examining parent-child physiological synchrony.

\section{Limitations and Future Research Directions}

Despite the initial contributions, our study is not without limitations. As mentioned earlier, although much research has linked parent-child synchrony with positive outcomes (e.g., Feldman et al. 2011), there is caveat warning that parent-child physiological synchrony might not always be a good sign (e.g., Papp et al. 2009). This is especially true under stressful situations such as maternal depression (Laurent et al. 2012) and children's exposure to interpartner violence (Hibel et al. 2009). Our study included a low-risk community sample with a comparatively large age range, so the maladaptive emotional parenting behaviors were generally low; more comprehensive research should be done to explore the potential association between parenting and parent-child physiological synchrony for specific at-risk populations. Also, given the cross-sectional nature of the current design, we could not determine the direction of relationship between emotional parenting behaviors and parent-child physiological synchrony. Additionally, the brain has recently been suggested as the fundamental platform for the development of synchrony, where oxytocin functionality affects both adult and child sociality (Feldman 2015). Future research should gather more neurophysiological evidence and explore the formation, development, and underlying processing mechanisms of parent-child synchrony during repeated daily interactions and investigate the role of parenting with consideration of neurophysiological synchrony.

Funding The author(s) disclosed receipt of the following financial support for the research, authorship, and/or publication of this article: this project was supported by the Faculty of Education, Beijing Normal University (Principal Investigator: Dr. Xiaoyi Hu 2018 Comprehensive Discipline Construction Fund).

Author contributions Z.R.H. designed and executed the study, assisted with the data analyses, and wrote the paper. M.M.G. collaborated with the design and writing of the study. J.Y. analyzed the data and 
wrote part of the results. X.H. and W.Z. collaborated with the design and writing of the study. X.L. collaborated in the writing and editing of the final manuscript.

\section{Compliance with ethical standards}

Conflict of interest The authors declare that they have no conflict of interest.

Ethical statement All procedures performed in studies involving human participants were in accordance with the ethical standards of the institutional and/or national research committee and with APA ethical standards.

Informed consent Informed consent was obtained from all individual participants included in the study (parents and children).

Publisher's note: Springer Nature remains neutral with regard to jurisdictional claims in published maps and institutional affiliations.

Open Access This article is distributed under the terms of the Creative Commons Attribution 4.0 International License (http://crea tivecommons.org/licenses/by/4.0/), which permits use, duplication, adaptation, distribution, and reproduction in any medium or format, as long as you give appropriate credit to the original author(s) and the source, provide a link to the Creative Commons license, and indicate if changes were made.

\section{References}

Achenbach, T. (1991). Manual for the Child Behavior Checklist/4 - 18 and 1991 Profile. Burlington, VT: University of Vermont Department of Psychiatry.

Barber, B.K., \& Harmon, E.L. (2002). Violating the self: parental psychological control of children and adolescents. Intrusive Parenting, 15-52. https://doi.org/10.1037/10422-002.

Berger, R. D., Akselrod, S., Gordon, D., \& Cohen, R. J. (1986). An efficient algorithm for spectral analysis of heart rate variability. IEEE Transactions on Bio-Medical Engineering, 33(9), 900-904.

Clearfield, M. W., Carter-Rodriguez, A., Merali, A. R., \& Shober, R. (2014). The effects of SES on infant and maternal diurnal salivary cortisol output. Infant Behavior and Development, 37(3), 298-304.

Crijnen, A. A., Achenbach, T. M., \& Verhulst, F. C. (1999). Problems reported by parents of children in multiple cultures: the child behavior checklist syndrome constructs. American Journal of Psychiatry, 156(4), 569-574.

Derogatis, L. R. (1994). Symptom Checklist-90-R: Administration, scoring, and procedures manual. SanAntonio, TX, Pearson.

Feldman, R. (2007). Parent-Infant Synchrony Biological Foundations and Developmental Outcomes. Current Directions in Psychological Science, 16(6), 340-345.

Feldman, R. (2012a). Bio-behavioral synchrony: a model for integrating biological and microsocial behavioral processes in the study of parenting. Parenting, 12, 154-164.

Feldman, R. (2012b). Parent-infant synchrony: a biobehavioral model of mutual influences in the formation of affiliative bonds. Monographs of the Society for Research in Child Development, 77(2), 42-51.

Feldman, R. (2015). Sensitive periods in human social development: new insights from research on oxytocin, synchrony, and high-risk parenting. Development and Psychopathology, 27(2), 369-395.
Feldman, R., \& Eidelman, A. I. (2004). Parent-infant synchrony and the social-emotional development of triplets. Developmental Psychology, 40(6), 1133-1147.

Feldman, R., Magori-Cohen, R., Galili, G., Singer, M., \& Louzoun, Y. (2011). Mother and infant coordinate heart rhythms through episodes of interaction synchrony. Infant Behavior and Development, 34(4), 569-577.

Fleming, A. S., O’Day, D. H., \& Kraemer, G. W. (1999). Neurobiology of mother-infant interactions: experience and central nervous system plasticity across development and generations. Neuroscience and Biobehavioral Reviews, 23(5), 673-685.

Graham, R. A., Scott, B. G., \& Weems, C. F. (2017). Parenting behaviors, parent heart rate variability, and their associations with adolescent heart rate variability. Journal of Youth and Adolescence, 46(5), 1089-1103.

Harrist, A. W., \& Waugh, R. M. (2002). Dyadic synchrony: its structure and function in children's development. Developmental Review, 22(4), 555-592.

Hibel, L. C., Granger, D. A., Blair, C., \& Cox, M. J. (2009). Intimate partner violence moderates the association between mother-infant adrenocortical activity across an emotional challenge. Journal of Family Psychology, 23(5), 615-25.

Laurent, H. K., Ablow, J. C., \& Measelle, J. (2012). Taking stress response out of the box: stability, discontinuity, and temperament effects on HPA and SNS across social stressors in mother-infant dyads. Developmental Psychology, 48, 35-45.

Leung, P. W. L., Kwong, S. L., Tang, C. P., Ho, T. P., Hung, S. F., \& Lee, C. C., et al. (2006). Test-retest reliability and criterion validity of the chinese version of CBCL, TRF, and YSR. Journal of Child Psychology and Psychiatry, 47(9), 970-973.

Lunkenheimer, E., Tiberio, S., Skoranski, A., Buss, K. A., \& Cole, P. M. (2017). Parent-child coregulation of parasympthetic processes varies by social context and risk for psychopathology. Psychophyisology, 55, e12985.

Papp, L. M., Pendry, P., \& Adam, E. K. (2009). Mother-adolescent physiological synchrony in naturalistic settings: within-family cortisol associations and moderators. Journal of Family Psychology, 23(6), 882-894.

Poston, D. L. J., \& Falbo, T. (1990). Scholastic and personality characteristics of only children and children with siblings in china. International Family Planning Perspectives, 16(2), $45-54$.

Repetti, R. L., Taylor, S. E., \& Seeman, T. E. (2002). Risky families: family social environments and the mental and physical health of offspring. Psychological Bulletin, 128(2), 330-366.

Rosenfeld, P., Melburg, V., \& Tedeschi, J. T. (1981). Forgetting as an impression management strategy in the forced-compliance situation. Journal of Social Psychology, 114(1), 69.

Shaffer, A., Yates, T. M., \& Egeland, B. R. (2009). The relation of emotional maltreatment to early adolescent competence: developmental processes in a prospective study. Child Abuse \& Neglect, 33(1), 36-44.

Suveg, C., Shaffer, A., \& Davis, M. (2016). Family stress moderates relations between physiological and behavioral synchrony and child self-regulation in mother-preschooler dyads. Developmental Psychobiology, 58(1), 83-97.

Williams, S. R., Cash, E., Daup, M., Geronimi, E. M. C., Sephton, S. E., \& Woodruff-Borden, J. (2013). Exploring patterns in cortisol synchrony among anxious and nonanxious mother and child dyads: a preliminary study. Biological Psychology, 93(2), 287-295.

Woltering, S., Lishak, V., Elliott, B., Ferraro, L., \& Granic, I. (2015). Dyadic attunement and physiological synchrony during motherchild interactions: an exploratory study in children with and without externalizing behavior problems. Journal of Psychopathology and Behavioral Assessment, 37(4), 624-633. 
Woody, M. L., Feurer, C., Sosoo, E. E., Hastings, P. D., \& Gibb, B. E. (2016). Synchrony of physiological activity during mother-child interaction: moderation by maternal history of major depressive disorder. Journal of Child Psychology and Psychiatry, 57(7), 843-850.
Zhang, Z. J. (2005). Handbook of behavioral medical scales. Chinese Journal of Behavioral Medical Science. Beijing: Chinese Medical Multimedia Press. 